\title{
LOCALLY COMPACT SPACES OF MEASURES
}

\author{
NORMAN Y. LUTHER
}

Abstract. Under varying conditions on the topological space $X$, the spaces of $\sigma$-smooth, $\tau$-smooth, and tight measures on $X$, respectively, are each shown to be locally compact in the weak topology if, and only if, $X$ is compact.

1. Introduction. Using the methods and results of Varadarajan [5], we give necessary and sufficient conditions on the topological space $X$ for the local compactness of the spaces of $\sigma$-smooth, $\tau$ smooth, and tight measures [resp., signed measures] on $X$, respectively, when endowed with the weak topology. For such spaces of signed measures, the finiteness of $X$ is easily seen to be necessary and sufficient (Remark 4). For such spaces of (nonnegative) measures, we find that, under various conditions on $X$, the compactness of $X$ is necessary and sufficient (Corollaries 3 and 4 ); in fact, it is the dividing line between when the space is locally compact and when it has no compact neighborhoods whatsoever (Theorems 1, 2, and 5).

2. Preliminaries and main results. Our definitions, notation, and terminology follow quite closely those in the paper of Varadarajan [5]. In particular, our topological definitions and terminology coincide with those of Kelley [3]. $X$ will denote a topological space and $C(X)$ the space of all real-valued, bounded, continuous functions on $X$. We use $B$ to denote the class of all zero-sets on $X$; i.e., $Z=\left\{f^{-1}(0)\right.$; $f \in C(X)\}$. The class of all co-zero sets (i.e., complements of zero-sets) in $X$ shall be denoted by $\mathfrak{U}$. The smallest algebra which contains $\mathbb{Z}$ shall be denoted by $\Im$.

A measure will mean a nonnegative, finite, finitely additive set function $m$ on $\Im$ such that for every $A \in \Im$,

$$
m(A)=\sup \{m(Z) ; A \supset Z \in \mathbb{Z}\} .
$$

The difference between any two measures is called a signed measure.

We shall let $\mathfrak{M}(X)=\{m ; m$ is a signed measure $\}$ and $\mathfrak{M}^{+}(X)$ $=\{m \in \mathfrak{M}(X) ; m \geqq 0\}$. We define, for each $m \in \mathfrak{M}(X)$, the norm

Received by the editors October 13, 1969.

AMS Subject Classifications. Primary 2813; Secondary 2830.

Key Words and Phrases. Finitely additive measures, signed measures, zero sets, co-zero sets, $\sigma$-smooth measures, $\tau$-smooth measures, tight measures, weak topology, locally compact spaces of measures, compact sets of measures, two-valued measures, degenerate measures, bounded continuous functions. 
$\|m\|=|m|(X)=m^{+}(X)+m^{-}(X)$, where $m^{+}, m^{-}$, and $|m|$ denote the positive, negative, and total variations of $m$, respectively. $\mathfrak{M}(X)$ with this norm is a Banach space. However, we shall use this norm only for descriptive purposes since we are interested in the weak topology (called the weak*-topology by some authors) on $\mathfrak{M}(X)$; this is the topology with basic neighborhoods of the form

$$
\begin{aligned}
& N\left(m_{0} ; f_{1}, \cdots, f_{n}, \epsilon\right) \\
& \quad=\left\{m \in \mathfrak{M}(X) ;\left|\int f_{i} d m-\int f_{i} d m_{0}\right|<\epsilon, i=1, \cdots, n\right\}
\end{aligned}
$$

where $m_{0} \in \mathfrak{M}(X),\left\{f_{1}, \cdots, f_{n}\right\}$ is any finite subset of $C(X)$, and $\epsilon>0$. A net $\left\{m_{\alpha}\right\}$ in $\mathfrak{M}(X)$ converges to $m \in \mathfrak{M}(X)$ in the weak topology if, and only if, $\int f d m_{\alpha} \rightarrow \int f d m$ for every $f \in C(X)$. $\mathfrak{M}(X)$ with the weak topology is a completely regular, Hausdorff, locally convex linear topological space and $\mathfrak{M}^{+}(X)$ is a closed subset [5, Theorem 1 , p. 181].

We shall be especially interested in certain subspaces of $\mathfrak{M}(X)$ endowed with the relativized weak topology. We describe them now. Let $m \in \mathfrak{M}(X) . m$ is $\sigma$-smooth if, and only if, $m\left(A_{n}\right) \rightarrow 0$ whenever $\left\{A_{n}\right\}$ is a sequence of sets in $\Im$ which decreases to $\varnothing . m$ is $\tau$-smooth if, and only if, $|m|\left(Z_{\alpha}\right) \rightarrow 0$ for any net $\left\{Z_{\alpha}\right\}$ of zero-sets for which (i) $Z_{\beta} \subset Z_{\alpha}$ if $\beta>\alpha$ and (ii) $\cap Z_{\alpha}=\varnothing$. Finally, $m$ is tight if, and only if, for any $\delta>0$ there exists a compact set $K \subset X$ such that $|m|_{*}(X-K)$ $<\delta$ where $|m|_{*}$ is defined for each $E \subset X$ by

$$
|m|_{*}(E)=\sup \{|m|(Z) ; E \supset Z \in \mathbb{Z}\} \text {. }
$$

(The definitions of $\tau$-smooth and tight here are not the same as in Varadarajan [5], but are equivalent [5, Theorem 24, p. 174, Theorem 29, p. 179].) We shall use $\mathfrak{M}_{\sigma}(X), \mathfrak{M}_{\tau}(X)$, and $\mathfrak{M}_{t}(X)$ to denote the sets of $\sigma$-smooth, $\tau$-smooth, and tight signed measures, respectively; $\mathfrak{M}_{\sigma}^{+}(X), \mathfrak{M}_{\tau}^{+}(X)$, and $\mathfrak{M}_{t}^{+}(X)$ will denote the spaces of $\sigma$-smooth, $\tau$-smooth, and tight measures, respectively. It is not hard to see that $\mathfrak{M}_{t}(X) \subset \mathfrak{M}_{\tau}(X) \subset \mathfrak{M}_{\sigma}(X) \subset \mathfrak{M}(X)$; hence also $\mathfrak{M}_{t}^{+}(X) \subset \mathfrak{M}_{\tau}^{+}(X)$ $\subset \mathfrak{M}_{\sigma}^{+}(X) \subset \mathfrak{M}^{+}(X)$. We note that $\mathfrak{M}_{\sigma}^{+}(X)$ is a closed subset of $\mathfrak{M}_{\sigma}(X)$ in the weak topology and that the same is true if $\sigma$ is replaced by $\tau$ or $t$.

A measure $m \in \mathfrak{M}^{+}(X)$ is two-valued if, and only if, (i) $m \neq 0$ and (ii) $m(A)$ is 0 or 1 for every $A \in \Im$. For each $x \in X$, we let $p_{x}$ denote the two-valued measure degenerate at the point $x$; i.e.,

$$
\begin{aligned}
p_{x}(A) & =1, \quad x \in A \\
& =0, \quad x \notin A
\end{aligned} \quad \text { for every } A \in \Im .
$$


We let $\mathfrak{T}^{+}(X)=\left\{m \in \mathfrak{M}^{+}(X) ; m\right.$ is two-valued $\}$ and $\mathfrak{D}(X)=\left\{p_{x}\right.$; $x \in X\}$. Clearly $\mathfrak{D}(X) \subset \mathfrak{M}_{t}^{+}(X)$ and $\mathfrak{D}(X) \subset \mathfrak{I}^{+}(X)$.

Each of the following remarks is either well known or easily verified.

REMARKs. 1. The norm-bounded subsets of $\mathfrak{M}(X)$ are relatively compact (i.e., their closures are compact) in the weak topology.

2. $\mathfrak{M}(X)$ is $\sigma$-compact and $\mathfrak{M}^{+}(X)$ is locally compact and $\sigma$-compact. This follows easily from Remark 1 and the fact that $\mathfrak{M}^{+}(X)$ is closed in $\mathfrak{M}(X)$.

3. If $X$ is compact, then $\mathfrak{M}(X)=\mathfrak{M}_{t}(X)\left(=\mathfrak{M}_{\sigma}(X)=\mathfrak{M}_{\tau}(X)\right)$. The converse is true if $X$ is completely regular and Hausdorff.

4. If $X$ is a completely regular, Hausdorff space, then the finiteness of $X$ is necessary and sufficient for the local compactness of any one of $\mathfrak{M}(X), \mathfrak{M}_{\sigma}(X), \mathfrak{M}_{\tau}(X)$, or $\mathfrak{M}_{t}(X)$. (For necessity, see [4, Theorem 7.8]; sufficiency follows from Remarks 3 and 1.)

5. If $X$ is a completely regular, Hausdorff space, then

(i) $\mathfrak{T}^{+}(X)$ is closed in $\mathfrak{M}(X)$,

(ii) $\mathfrak{I}^{+}(X) \cap \mathfrak{M}_{\tau}^{+}(X)=\mathfrak{I}^{+}(X) \cap \mathfrak{M}_{t}^{+}(X)=\mathfrak{D}(X)$, and

(iii) $T(x)=p_{x}$ defines a homeomorphism of $X$ onto $\mathfrak{D}(X)[\mathbf{5}$, Theorem 11, p. 187, Corollary 3, p. 175, Theorem 9, p. 187].

6. A set $F \subset \mathfrak{M}_{\sigma}^{+}(X)$ is relatively compact in $\mathfrak{M}_{\sigma}^{+}(X)$ if, and only if, for any sequence $\left\{g_{j}\right\}$ in $C(X)$ which decreases to zero, $\int g_{j} d m \rightarrow 0$ uniformly in $m \in F$ [5, Theorem 25(2), p. 200].

We shall also use the fact [5, Remark 3, p. 183] that the family of all sets of the form

$$
\begin{aligned}
& N^{*}\left(m_{0} ; U_{1}, \cdots, U_{k}, \epsilon\right) \\
& =\left\{m \in \mathfrak{M}^{+}(X) ; m\left(U_{i}\right)-m_{0}\left(U_{i}\right)>-\epsilon\right. \\
& \left.\quad \text { for all } i=1, \cdots, k, \text { and }\left|m(X)-m_{0}(X)\right|<\epsilon\right\}
\end{aligned}
$$

where $m_{0} \in \mathfrak{M}^{+}(X), U_{1}, \cdots, U_{k} \in \mathfrak{U}$, and $\epsilon>0$ form a basis for the neighborhoods of the weak topology on $\mathfrak{M}^{+}(X)$.

We shall now state and prove our main results. We recall that any metric space is paracompact.

Theorem 1. Let $X$ be a paracompact, Hausdorff topological space.

(I) If $X$ is compact, then $\mathfrak{M}_{\sigma}^{+}(X)$ is locally compact and $\sigma$-compact.

(II) If $X$ is not compact, then $\mathfrak{M}_{\sigma}^{+}(X)$ has no compact neighborhoods whatsoever.

REMARK. We can weaken our hypothesis on $X$ in Theorem 1 (and in Corollary 4 below) to metacompact, normal, and Hausdorff (see $[3,5 \mathrm{~V}(\mathrm{c})])$ or to realcompact, normal, and Hausdorff.

ProOF. (I) follows from Remarks 2 and 3. For the proof of (II), we let $m_{0} \in \mathfrak{M}_{\sigma}^{+}(X)$ and consider a basic neighborhood 


$$
V=N^{*}\left(m_{0} ; U_{1}, \cdots, U_{k}, \epsilon\right) \cap \mathfrak{M}_{\sigma}^{+}(X) .
$$

There is a sequence $\left\{x_{n}\right\} \subset X$ which has no cluster point since $X$ is paracompact, Hausdorff and is not compact. Let $m_{n}=m_{0}+(\epsilon / 2) p_{x_{n}}$, $n=1,2, \cdots$. Then clearly $\left\{m_{n}\right\} \subset V$. To show $V$ is not relatively compact in $\mathfrak{M}_{\sigma}^{+}(X)$, it suffices to show that $\left\{m_{n}\right\}$ is not relatively compact in $\mathfrak{M}_{\sigma}^{+}(X)$.

We define $F_{n}=\left\{x_{m} ; m \neq n\right\}, n=1,2, \cdots$. Since $\left\{x_{n}\right\}$ has no cluster point, $F_{n}$ is closed for every $n$. Consequently, by the normality of $X$ there are open sets $U_{n}$ and closed sets $Y_{n}$ such that $x_{n} \in U_{n}$ $\subset Y_{n} \subset X-F_{n}$ for every $n$. Define $V_{n}=U_{n}-\bigcup_{i=1}^{n-1} Y_{i}, n=1,2, \cdots$. The open sets $V_{n}$ are disjoint and $x_{n} \in V_{n}$ for every $n$. We define $H_{k}=X-\cup_{n \geqq k} V_{n}, k=1,2, \cdots$. Then $\left\{H_{k}\right\}$ is an increasing sequence of closed sets whose union is $X$. Moreover, $H_{k}$ and $\left\{x_{k}, x_{k+1}, \cdots\right\}$ are disjoint closed sets so that, by the normality of $X$, there are continuous functions $h_{k}: X \rightarrow[0,1]$ such that $h_{k}$ is 0 on $H_{k}$ and is 1 on $\left\{x_{k}, x_{k+1}, \cdots\right\}$. If we define $g_{j}=\min \left\{h_{1}, \cdots, h_{j}\right\}, j=1,2, \cdots$, one can readily see that $g_{j} \in C(X), g_{j}$ decreases to the zero function, and $g_{j}=1$ on $\left\{x_{j}, x_{j+1}, \cdots\right\}$. But, consequently, if $n \geqq j$, then $\int g_{j} d m_{n} \geqq \epsilon / 2$. Hence by Remark $6\left\{m_{n}\right\}$ is not relatively compact.

ExAmple. If $X$ is pseudo-compact (i.e., every continuous function on $X$ is bounded), then $\mathfrak{M}_{\sigma}(X)=\mathfrak{M}(X)([\mathbf{1}],[5$, Theorem 21, p. 172]) and, consequently, $\mathfrak{M}_{\sigma}^{+}(X)$ is locally compact (Remark 2 ). Since there is a countably compact (hence pseudo-compact), locally compact, first countable, normal, Hausdorff space which is not compact [3, $5 \mathrm{E}(\mathrm{e})]$, we cannot hope, in either Theorem 1 or Corollary 4 (below), to significantly weaken the hypotheses on $X$ beyond that remarked above.

THEOREM 2. Let $X$ be a completely regular, Hausdorff space.

(I) If $X$ is compact, then $\mathfrak{M}_{t}^{+}(X)\left(=\mathfrak{M}_{\tau}^{+}(X)\right)$ is locally compact and $\sigma$-compact.

(II) If $X$ is not compact, then neither $\mathfrak{M}_{t}^{+}(X)$ nor $\mathfrak{M}_{\tau}^{+}(X)$ has any compact neighborhoods whatsoever.

Proof. The proof of (II) is the same for $\mathfrak{M}_{\tau}^{+}(X)$ and $\mathfrak{M}_{t}^{+}(X)$ so we restrict ourselves to the latter case. Let $m_{0} \in \mathfrak{M}_{t}^{+}(X)$ and consider $V=N^{*}\left(m_{0} ; U_{1}, \cdots, U_{k}, \epsilon\right) \cap \mathfrak{M}_{t}^{+}(X)$. Since $X$ is not compact there is a net $\left\{x_{\alpha}\right\}_{\alpha \in A}$ in $X$ which has no cluster point in $X$. Define $m_{\alpha}=m_{0}$ $+(\epsilon / 2) p_{x_{\alpha}}$ for every $\alpha \in A$. Clearly $\left\{m_{\alpha}\right\}_{\alpha \in A} \subset V$. Thus it suffices to show that the closure of $\left\{m_{\alpha}\right\}_{\alpha \in A}$ in $\mathfrak{M}_{t}^{+}(X)$ is not compact. This set is compact if, and only if, the closure of $\left\{p_{x_{\alpha}}\right\}_{\alpha \in A}$ in $\mathfrak{M}_{t}^{+}(X)$ is compact. But this, in turn, is equivalent to the compactness of the closure of $\left\{p_{x_{\alpha}}\right\}_{\alpha \in A}$ in $\mathfrak{D}(X)$ because $\mathfrak{D}(X)$ is closed in $\mathfrak{M}_{t}^{+}(X)$ (Remark 5 
(i), (ii)). Equivalently, by Remark 5 (iii), the closure of $\left\{x_{\alpha}\right\}_{\alpha \in A}$ in $X$ is compact. But this leads to a contradiction since $\left\{x_{\alpha}\right\}_{\alpha \in A}$ has no cluster point in $X$.

COROLlaRY 3. Let $X$ be completely regular and Hausdorff. The following are equivalent:

(a) $X$ is compact.

(b) $\mathfrak{M}_{\tau}^{+}(X)$ is locally compact.

(c) $\mathfrak{M}_{t}^{+}(X)$ is locally compact.

The preceding corollary and Theorem 1 yield:

CoRollary 4. Let $X$ be a paracompact, Hausdorff space. The following are equivalent:

(a) $X$ is compact.

(b) $\mathfrak{M}_{\sigma}^{+}(X)$ is locally compact.

(c) $\mathfrak{M}_{\tau}^{+}(X)$ is locally compact.

(d) $\mathfrak{M}_{t}^{+}(X)$ is locally compact.

The following result is contained in Theorem 2 if $X$ is Hausdorff. We omit the proof which begins in the same manner as that of Theorem 2 and ends with the use of [5, Theorem 29, p. 205].

THEOREM 5. Let $X$ be a locally compact, regular space such that $\mathfrak{M}_{\sigma}^{+}(X)=\mathfrak{M}_{t}^{+}(X)$.

(I) If $X$ is compact, then $\mathfrak{M}_{\sigma}^{+}(X)\left(=\mathfrak{M}_{\tau}^{+}(X)=\mathfrak{M}_{t}^{+}(X)\right)$ is locally compact and $\sigma$-compact.

(II) If $X$ is not compact, then $\mathfrak{M}_{\sigma}^{+}(X)\left(=\mathfrak{M}_{\tau}^{+}(X)=\mathfrak{M}_{t}^{+}(X)\right)$ has no compact neighborhoods whatsoever.

REMARK. Varadarajan [5, Remark, p. 205] gives conditions under which the hypotheses of Theorem 5 are fulfilled. One instance is when $X$ is locally compact, regular, and $\sigma$-compact.

EXAmple. Let $X$ be a regular, Hausdorff, noncompact space such that every real-valued continuous function on $X$ is constant [2]. Clearly $\mathfrak{M}(X)=\mathfrak{M}_{\sigma}(X)=\mathfrak{M}_{\tau}(X)=\mathfrak{M}_{t}(X)$ is locally compact, thus so is $\mathfrak{M}^{+}(X)=\mathfrak{M}_{\sigma}^{+}(X)=\mathfrak{M}_{\tau}^{+}(X)=\mathfrak{M}_{t}^{+}(X)$. Consequently, we cannot hope to significantly weaken the hypotheses on $X$ in Remark 4, in Theorems 2 or 5 , or in Corollary 3.

3. Complements; probability measures. Let $P_{\sigma}(X)=\left\{m \in \mathfrak{M}_{\sigma}^{+}(X)\right.$; $m(X)=1\}, \quad P_{\tau}(X)=\left\{m \in \mathfrak{M}_{\tau}^{+}(X) ; \quad m(X)=1\right\}, \quad$ and $P_{t}(X)$ $=\left\{m \in \mathfrak{M}_{t}^{+}(X) ; m(X)=1\right\}$, each endowed with the relativized weak topology. Theorems 1,2 , and 5 and Corollaries 3 and 4 all remain valid if $\mathfrak{M}_{\sigma}^{+}(X), \mathfrak{M}_{\tau}^{+}(X)$, and $\mathfrak{M}_{t}^{+}(X)$ are replaced by $P_{\sigma}(X), P_{\tau}(X)$, and $P_{t}(X)$, respectively. For in the proof of Theorem 1, along with 
replacing $\mathfrak{M}_{\sigma}^{+}(X)$ by $P_{\sigma}(X)$, one must simply change the definition of $m_{n}$ to $m_{n}=(1-\epsilon / 2) m_{0}+(\epsilon / 2) p_{x_{n}}(0<\epsilon \leqq 2)$; and similarly in the proofs of Theorems 2 and 5 . Then these proofs will apply since $P_{\sigma}(X), P_{\tau}(X)$, and $P_{t}(X)$ are closed in $\mathfrak{M}_{\sigma}^{+}(X), \mathfrak{M}_{\tau}^{+}(X)$, and $\mathfrak{M}_{t}^{+}(X)$, respectively.

Now if $X$ is a completely regular, Hausdorff space, then $\mathfrak{D}(X)$ is closed in each of $\mathfrak{M}_{\tau}^{+}(X), \mathfrak{M}_{t}^{+}(X)$, their respective closed unit balls, $P_{\tau}(X)$, and $P_{t}(X)$. Hence $X$ will share any topological properties of these spaces which are inherited by closed subspaces (Remark 5 (iii)). Normality, paracompactness, compactness, and $\sigma$-compactness are examples. This observation verifies the implications $(b) \Rightarrow(a)$, (c) $\Rightarrow(a),(d) \Rightarrow(a)$, and (e) $\Rightarrow(a)$ of our final result, which must be well known. The reverse implications follow from Remarks 3 and 1.

Theorem 6. Let $X$ be a completely regular, Hausdorff space. The following are equivalent:

(a) $X$ is compact.

(b) The closed unit ball $\left\{m \in \mathfrak{M}_{\tau}^{+}(X) ;\|m\| \leqq 1\right\}$ in $\mathfrak{M}_{\tau}^{+}(X)$ is compact.

(c) The closed unit ball $\left\{m \in \mathfrak{M}_{t}^{+}(X) ;\|m\| \leqq 1\right\}$ in $\mathfrak{M}_{t}^{+}(X)$ is compact.

(d) $P_{\tau}(X)$ is compact.

(e) $P_{t}(X)$ is compact.

It follows that if $X$ is a completely regular, Hausdorff space, then $P_{\tau}(X)$ [resp., $P_{t}(X)$ ] is locally compact if, and only if, it is compact. Clearly the same result holds for $P_{\sigma}(X)$ if $X$ is a normal, Hausdorff space.

4. Added in proof. The following result generalizes Theorem 1.

THEOREM $1^{\prime}$. Let $X$ be a topological space.

(I) If $X$ is pseudo-compact, then $\mathfrak{M}_{\sigma}^{+}(X)$ is locally compact and $\sigma$-compact.

(II) If $X$ is not pseudo-compact, then $\mathfrak{M}_{\sigma}^{+}(X)$ has no compact neighborhoods whatsoever.

Proof. (I) follows from Remark 2. For (II) we let $m_{0} \in \mathfrak{M}_{\sigma}^{+}(X)$ and consider $V=N^{*}\left(m_{0}, U_{1}, \cdots, U_{k, \epsilon}\right) \cap \mathfrak{M}_{\sigma}^{+}(X)$. Since $X$ is not pseudocompact, there is a regular sequence $\left\{Z_{n}\right\}$ of zero-sets in $X$ such that $X-Z_{n} \neq \varnothing$ for every $n\left[5\right.$, Theorem 16, p. 170]. Let $x_{n} \in X-Z_{n}$ and define $m_{n}=m_{0}+(\epsilon / 2) p_{x_{n}}, n=1,2, \cdots$. Then clearly $\left\{m_{n}\right\} \subset V$. But $m_{n}\left(X-Z_{n}\right) \geqq \epsilon / 2$ for every $n$ so that $V$ is not relatively compact [5, Theorem 25 (1), p. 200]. 
As before, Theorem $1^{\prime}$ remains valid if $\mathfrak{M}_{\sigma}^{+}(X)$ is replaced by $P_{\sigma}(X)$. Consequently, if $X$ is any topological space, then $P_{\sigma}(X)$ is locally compact if, and only if, it is compact.

\section{REFERENCES}

1. I. Glicksberg, The representation of functionals by integrals, Duke Math. J. 19 (1952), 253-261. MR 14, 288.

2. E. Hewitt, On two problems of Urysohn, Ann. of Math. (2) 47 (1946), 503-509. MR 8, 165.

3. J. L. Kelley, General topology, Van Nostrand, Princeton, N. J., 1955. MR 16, 1136.

4. J. L. Kelley and I. Namioka, Linear topological spaces, The University Series in Higher Math., Van Nostrand, Princeton, N. J., 1963. MR 29 \#3851.

5. V. S. Varadarajan, Measures on topological spaces, Mat. Sb. 55 (97) (1961), 33-100; English transl., Amer. Math. Soc. Transl. (2) 48 (1965), 161-228. MR 26 $\# 6342$.

Washington State University, Pullman, Washington 99163 